\title{
How Histone Modifications Change Nucleosome Stability - FRET Studies on Single Molecules and in Bulk
}

\author{
Katalin Tóth ${ }^{1}$, Alexander Gansen ${ }^{1}$, Szabolcs Hetey ${ }^{2}$, Loránd Székvölgyi ${ }^{2}$, Lars Nordenskiöld ${ }^{3}$, Jörg \\ Langowski ${ }^{1}$ \\ 1 . German Cancer Research Center, Heidelberg, Germany \\ 2 . University of Debrecen, Hungary \\ ${ }^{3}$. Nanyang Technological University, Singapore
}

Histones, once regarded as highly conserved genome-compacting proteins, today show their role as active players in transcription. Posttranslational modifications and point mutations of histones are related to different genetic activities and pathologies. We are looking for the consequences of these chemical modifications at the level of the structure of single, reconstituted nucleosomes.

Using Förster resonance energy transfer (FRET) measurements in bulk and on single molecules, we assessed the structural role of histone acetylation and of some point mutations in nucleosomes reconstituted on the 170bp long Widom 601 DNA sequence. By measuring distances between fluorescently labeled parts of the nucleosome we could follow the salt-induced nucleosome disassembly, using donor-acceptor pairs on the ends or in the internal part of the nucleosomal DNA, and on histones. The single molecule observations helped to characterize the intermediate states as well as the distributions of the populations $[1,2]$.

We have demonstrated earlier [3], that in the absence of posttranslational modifications the nucleosome stability is only slightly influenced by the origin of the histones (Xenopus laevis, mice, yeast). On the other hand, the DNA sequence has an important effect on the stability: nucleosomes prepared with one of the best positioning natural sequences (Xenopus borealis 5S rDNA) disintegrate at much lower salt concentrations than those with the known best positioning sequence, the SELEX selected "601".

Histone hyperacetylation is often related to enhanced gene transcription and local decompaction of the chromatin. To analyze the structural effect of acetylation, we applied chemical acetylation of the different recombinant histones. This results in a statistical distribution of acetylated lysines. At the nucleosomal level we observed that the structural effect of acetylation of the different histones is not cumulative. Earlier bulk FRET measurements with end-labeled DNA have shown that at physiological salt concentrations acetylation of only the $\mathrm{H} 3$ histones alone opens the linker DNA while acetylation of only the $\mathrm{H} 4$ histones leads to compaction. [4]. Our present data allows us to distinguish two different effects of acetylation at two stages of salt-induced nucleosome destabilization: linker DNA unwrapping, and disintegration with dimer release. Nucleosome unwrapping was monitored by FRET between the linker ends of the DNA, while FRET experiments between labels positioned on the internal part of the nucleosome reported on nucleosome disassembly. We detect enhanced DNA unwrapping above physiological salt concentrations upon acetylation of either $\mathrm{H} 4$ or $\mathrm{H} 3$. On the other hand, complete disintegration with dimer release is enhanced only in the case of $\mathrm{H3}$ acetylation; acetylation of $\mathrm{H} 4$ even counteracts this process. This result suggests that $\mathrm{H} 3$ and $\mathrm{H} 4$ acetylation have partially opposing effects in regulating nucleosome architecture and that distinct aspects of nucleosome dynamics might be independently controlled by selective histone acetylation. 
Posttranslational modifications or single amino acid mutations in chromatin gain more and more attention. We are investigating the role of such modifications using the same methods, looking for the effects of these small structural variations on the stability of the nuclesosome. We report about the effect of the acetylation of lysine 16 in the tail of histone H4, and on the mutation of lysine 27 in the H3 tail. Regardless of the state of acetylation, origin of histones or DNA sequences nucleosomes disassemble via an intermediate state which is suppressed at higher nucleosome concentration, confirming our proposed model of step-wise disassembly.

\section{References:}

[1] A. Gansen, A. Valeri, F. Hauger, S. Felekyan, S. Kalinin, K. Tóth, J. Langowski, C. Seidel (2009): Nucleosome disassembly intermediates characterized by single-molecule FRET. Proc. Natl. Acad. Sci. USA 11(45), 15308-13

[2] Böhm,V., Gansen,A., Hieb, A., Tóth, K., Andrews, A., Luger, K., Langowski, J. (2011): Nucleosomal DNA accessibility governed by the dimer/tetramer interface. Nucleic Acids Research 39 (8): 3093-102.

[3] Tóth K, Böhm V. Sellmann C, Danner M, Hanne J, Berg M, Barz I, Gansen A, Langowski J (2013) Histone- and DNA sequence-dependent stability of nucleosomes studied by single pair FRET.Cytometry A 83A, 839-846.

[4] Tóth K, Brun N, Langowski J(2006): Chromatin compaction at the mononucleosome level. Biochemistry, 45, 1591-1598 